\title{
ON THE NUMBER OF TOPOLOGIES DEFINABLE FOR A FINITE SET
}

\author{
A. SHAFAAT
}

(Received 6 July 1966)

No general rule for determining the number $N(n)$ of topologies definable for a finite set of cardinal $n$ is known. In this note we relate $N(n)$ to a function $F_{t}\left(r_{1}, \cdots, r_{t+1}\right)$ defined below which has a simple combinatorial interpretation. This relationship seems useful for the study of $N(n)$. In particular this can be used to calculate $N(n)$ for small values. For $n=3,4,5,6$ we find $N(3)=29, N(4)=355, N(5)=7,181, N(6)=145,807$.

Let $T$ be a topology on a finite set $E$. Let $S_{1}$ be the collection of all non-empty sets in $T$ which do not properly coritain any non-empty set in $T$. It is clear that $S_{1}$ is a collection of disjoint subsets of $E$. If for any collection $K$ of sets $P_{U}(K)$ denotes the set of all non-empty unions of sets in $K$ then $P_{\cup}\left(S_{1}\right) \subseteq T$. Let $\cup S_{1}$ be the union of all sets in $S_{1}$. Then every non-empty set in $T$ is of the form $U \cup V$ where $V \in P_{U}\left(S_{1}\right)$ and $U$ is a subset of $E-\cup S_{1}$. Let $T_{1}$ be the collection of all the sets $U$ and the null set. It can be easily proved that $T_{1}$ is a topology on $E-\cup S_{1}$. We shall refer to $S_{1}$ and $T_{1}$ as "nucleus" and "orbital topology" of the topology $T$, respectively.

By a "reduced base" of a topology on a finite set we shall mean a base such that no base set is a union of other base sets.

THEOREM. Let $B_{1}$ be a reduced base for $T_{1}$. Then there is a unique singlevalued mapping $f: B_{1} \rightarrow P_{\cup}\left(S_{1}\right)$ such that $B=\left\{X_{1} \cup X_{1} f, X_{1} \in B_{1}\right\} \cup S_{1}$ is a reduced base for $T$. Also, $f$ preserves the inclusion relation $\subseteq$ for sets. Conversely if $S_{1}$ is a non-empty collection of disjoint non-empty subsets of $E$, $T_{1}$ is any topology on $E-\cup S_{1}$ and $f$ is a single-valued mapping from a reduced base $B_{1}$ for $T_{1}$ into $P_{\cup}\left(S_{1}\right)$ which preserves $\subseteq$ then $B=\left\{X_{1} \cup X_{1} f\right.$, $\left.X_{1} \in B_{1}\right\} \cup S_{1}$ is a reduced base for a topology $T$ on $E$ such that $S_{1}, T_{1}$ are respectively the nucleus and the orbital topology of $T$.

Proof. For any $X_{1} \in B_{1}$, we define $X_{1} f$ to be a member of $P_{\cup}\left(S_{1}\right)$ such that $X_{1} \cup X_{1} f \in T$ and $X_{1} \cup V \notin T$ if $X_{1} f \supset V . X_{1} f$ exists because $T_{1}$ is the orbital topology of $T$. If $V^{*} \in P_{U}\left(S_{1}\right)$ has the property stated for $X_{1} f$ then $V^{*} \supseteqq X_{1} f$ and $X_{1} f \supseteqq V^{*}$, so that $X_{1} f=V^{*}$. Thus $f$ is a mapping from $B_{1}$ into $P_{\cup}\left(S_{1}\right)$. We show that $f$ is the mapping required by the first 
part of the theorem. Let $X_{1} \subseteq X_{1}^{\prime}$; then

$$
\left(X_{1} \cup X_{1} f\right) \cap\left(X_{1}^{\prime} \cup X_{1}^{\prime} f\right)=X_{1} \cup\left(X_{1} f \cap X_{1}^{\prime} f\right) \in T,
$$

since $X_{1}, X_{1}^{\prime} f$ are disjoint for all $X_{1}, X_{1}^{\prime} \in B_{1}$. We conclude from the definition of $f$ that $X_{1} f \cap X_{1}^{\prime} f=X_{1} f$ so that $X_{1} f \subseteq X_{1}^{\prime} f$ and hence $f$ preserves $\cong$. Next let $Y \in T$ and let $Y=U \cup V$, where $U \in T_{1}, V \in P_{U}\left(S_{1}\right)$. Since $B_{1}$ is a base for $T_{1}$ we can write $U=\cup B_{1}^{\prime}$ for some subcollection $B_{1}^{\prime}$ of $B_{1}$. If $U$ is empty, $Y$ is trivially a union of sets in

$$
B=\left\{X_{1} \cup X_{1} f, X_{1} \in B_{1}\right\} \cup S_{1} .
$$

Hence we can suppose $B_{1}^{\prime}$ non-empty. Then $X_{1}^{\prime} f \subseteq V$ for every $X_{1}^{\prime} \in B_{1}^{\prime}$; for

$$
X_{1}^{\prime} \cup\left(V \cap X_{1}^{\prime} f\right)=(U \cup V) \cap\left(X_{1}^{\prime} \cup X_{1}^{\prime} f\right) \in T
$$

and therefore $V \cap X_{1}^{\prime} f=X_{1}^{\prime} f$. Hence $Y=\cup\left\{X_{1}^{\prime} \cup X_{1}^{\prime} f, X_{1}^{\prime} \in B_{1}^{\prime}\right\} \cup$ (union of sets in $S_{1}$ ). This proves that $B$ is a base for $T$. That $B$ is reduced follows directly from the definition of $f$ and the assumption that $B_{1}$ is reduced. To prove the uniqueness of the mapping $f$ suppose that $f^{*}$ is another mapping satisfying the first part of the theorem. Then, for some $X_{1} \in B_{1}, X_{1} f \subset X_{1} f^{*}$. But $X_{1} \cup X_{1} f \in T$ and therefore is a union of sets in $B^{*}=\left\{Y_{1} \cup Y_{1} f^{*}, Y_{1} \in B_{1}\right\} \cup S_{1}$. Since $B_{1}$ is reduced this is impossible in view of $X_{1} f \subset X_{1} f^{*}$.

For the converse, let $B$ be as defined in the theorem. Then $E=\cup B=\left(\cup B_{1}\right) \cup\left(\cup S_{1}\right)$. Let $Y, Y^{*}$ be any two members of $B$ and write $Y=X_{1} \cup X_{1} f, Y^{*}=X_{1}^{*} \cup X_{1}^{*} f$. Since $f$ preserves $\subseteq$,

$$
\begin{aligned}
Y \cap Y^{*} & =\left(X_{1} \cap X_{1}^{*}\right) \cup\left(X_{1} f \cap X_{1}^{*} f\right) \\
& \left.=\left(X_{1} \cap X_{1}^{*}\right) \cup\left(X_{1} \cap X_{1}^{*}\right) f \cup \text { (union of sets in } S_{1}\right) .
\end{aligned}
$$

Now $X_{1}, X_{1}^{*} \in B_{1}$ and $X_{1} \cap X_{1}^{*}=\cup B_{1}^{\prime}$, where $B_{1}^{\prime}$ is a subcollection of $B_{1}$. Since $Z_{1}^{\prime} f \subseteq\left(X_{1} \cap X_{1}^{*}\right) f$ for every $Z_{1}^{\prime} \in B_{1}^{\prime}$, this gives

$$
Y \cap Y^{*}=\cup\left\{Z_{1}^{\prime} \cup Z_{1}^{\prime} f, Z_{1}^{\prime} \in B_{1}^{\prime}\right\} \cup \text { (union of members of } S_{1} \text { ); }
$$

so that $Y \cap Y^{*}$ is a union of members of $B$. In case one or both of $Y, Y^{*}$ are members of $S_{1}$ and therefore not expressible in the form $X \cup X f$, $Y \cap Y^{*}$ is trivially a union of sets in $B$. Hence the intersection of any two members of $B$ is a union of members of $B$ and therefore $B$ is a base for a topology $T$ on $E$. The rest of the theorem now follows directly.

For any topology $T$ on a finite set $E$ we can form the sequence $T_{0}=T$, $\left(S_{1}, T_{1}\right),\left(S_{2}, T_{2}\right), \cdots,\left(S_{t}, T_{t}\right), S_{t+1}$, where $S_{k}, T_{k}$ are respectively the nucleus and the orbital topology of $T_{k-1}$ for $t \geqq k \geqq 1$ and $S_{t+1}$ is a reduced base as well as the nucleus of $T_{t}$, so that $T_{t}=P_{U}\left(S_{t+1}\right)$. By the above theorem there is a unique sequence of mappings $f_{1}, \cdots, f_{t}$ such that for 
$\mathbf{1} \leqq i \leqq t, f_{i}$ maps $B_{i}$ into $P_{U}\left(S_{i}\right)$, where $B_{i}$ is a reduced base for $T_{i}$ and is defined by

$$
\begin{array}{r}
B_{t}=S_{t+1}, B_{i}=\left\{X_{i+1} \cup X_{i+1} f_{i+1}, X_{i+1} \in B_{i+1}\right\} \cup S_{i+1}, \\
\text { for } 0 \leqq i \leqq t .
\end{array}
$$

By our theorem, every topology on $E$ can be obtained as follows: Partition $E$ into any number, say $r$, of disjoint and collectively exhaustive classes $E_{1}, \cdots, E_{r}$ and then partition, in an arbitrary way, the set $\left\{E_{1}, \cdots, E_{r}\right\}$ into disjoint and collectively exhaustive classes, say, $S_{1}, \cdots, S_{t+1}$. Let $f_{1}, \cdots, f_{t}$ be any mappings such that

(i) $f_{t}$ maps $B_{t}=S_{t+1}$ into $P_{U}\left(S_{t}\right)$,

(ii) $f_{t-i}$ maps $B_{t-i}$ into $P_{U}\left(S_{t-i}\right)$ where

$$
B_{t-i}=\left\{X \cup X f_{t-i+1}, X \in B_{t-i+1}\right\} \cup S_{t-i+1},
$$

(iii) each of the mappings $f_{1}, \cdots, f_{t}$ preserves the inclusion relation $\subseteq$ for sets.

Then $B=B_{0}=\left\{X_{1} \cup X_{1} f_{1}, X_{1} \in B_{1}\right\} \cup S_{1}$ is a base for a topology on $E$ and every topology on $E$ is obtained in this way.

In view of this we can express the number $N(n)$ of topologies definable for a finite set of cardinal $n$ as follows:

$$
N(n)=\sum_{r=1}^{n}\left[M_{n, r} r ! \sum_{r_{1}+\cdots r_{t+1}=r}\left\{\left[F_{t}\left(r_{1}, \cdots, r_{t+1}\right) / r_{1} ! \cdots r_{t+1} !\right]\right\}\right]
$$

where $M_{n, r}$ is the number of ways a set of order $n$ can be partitioned into $r$ unordered classes and $F_{t}\left(r_{1}, \cdots, r_{t+1}\right)$ is the number of sequences of mappings $f_{1}, \cdots, f_{t}$ described above when $S_{1}, \cdots, S_{t+1}$ have $r_{1}, \cdots, r_{t+1}$ members respectively. The summation in curly brackets extends over all finite sequences $r_{1}, \cdots, r_{t+1}$ of positive integers satisfying $r_{1}+\cdots+r_{t+1}=r$.

The following recurrence relation holds for $M_{n, r}$ :

\begin{tabular}{|c|c|c|c|c|c|c|c|}
\hline \multirow[t]{3}{*}{$x(1,1)$} & \multirow[t]{2}{*}{$x\left(1, e_{j}\right)$} & & & & & & \\
\hline & & $x\left(2, e_{1}+1\right)$ & $x\left(2, e_{2}\right)$ & & & & \\
\hline & & & & $x\left(3, e_{2}+1\right)$ & $x\left(\mathbf{3}, e_{3}\right)$ & & \\
\hline & & & & & & $x\left(4, e_{3}+1\right)$ & $x\left(4, e_{4}\right)$ \\
\hline
\end{tabular}

$$
M_{n+1, r}=r M_{n, r}+M_{n, r-1} .
$$

The function $F_{t}\left(r_{1}, \cdots, r_{t+1}\right)$ has a simple combinatorial interpretation which we explain by taking $t=3$ and by referring to the figure below.

Figure 1 
In this figure we have taken $e_{1}=r_{4}, e_{2}=r_{3}+r_{4}, e_{3}=r_{2}+r_{3}+r_{4}$, $e_{4}=r_{1}+r_{2}+r_{3}+r_{4}$. Every one of the $r_{4}$ squares in the first row is given to be occupied with just one of the symbols $x(1,1), \cdots, x\left(1, e_{1}\right)$ that are labels for sets in $S_{4}$. In the second row only the last $r_{3}$ squares on the right are given to be initially occupied, each by just one of the $r_{3}$ symbols $x\left(2, e_{1}+1\right), \cdots, x\left(2, e_{2}\right)$ that similarly stand for sets in $S_{3}$; and so on. Let us refer to the $j$ th square from the left in the $i$ th row from the top as $\sigma(i, j)$. In what follows we shall not explicitly mention the restrictions on the ranges of the variables $i, j, k, \cdots$. Write $\Sigma(i, j)=\{x(i, j)\}$ if $\sigma(i, j)$ is not initially empty. The combinatorial problem now is to place in every empty square $\sigma(i, j)$ a non-empty set $\Sigma(i, j)$ of symbols such that

(iv) $\Sigma(i, j) \cong\left\{x\left(i, e_{i-1}+1\right), \cdots, x\left(i, e_{i}\right)\right\}$,

(v) $\quad x(i, k) \in \Sigma(i, j)$ implies $\Sigma(i+1, k) \subseteq \Sigma(i+1, j)$.

Thus, for example, the conditions (iv), (v) compel us to place in the empty squares of the third row in Fig. 1 symbols chosen from $x\left(3, e_{2}+1\right), \cdots, x\left(3, e_{3}\right)$, and if $x\left(3, e_{3}\right)$ has been placed in $\sigma\left(3, e_{2}\right)$ (the square immediately below the one containing $\left.x\left(2, e_{2}\right)\right)$ then $x\left(3, e_{3}\right)$ will have to occur in any set of symbols to be placed in a square of the third row which comes directly under a square containing $x\left(2, e_{2}\right)$. Let $Y(i, k)=\bigcup_{l=1}^{i} \Sigma(l, k)$. Then it is easily seen that if we let $B_{4-i}$ be the set of all $Y(i, k)$ for fixed $i$ and write $Y(i, k) f_{4-i}=\Sigma(i+1, k)$ then $B_{4-i}, f_{4-i}$ satisfy (i), (ii), (iii) for $t=3 .^{1} \mathrm{It}$ follows that $F_{3}\left(r_{1}, r_{2}, r_{3}, r_{4}\right)$ is the number of ways of placing the symbols $x(i, j)$ in the empty squares of Fig. 1 such that (iv) and (v) are satisfied.

We can use this interpretation of $F_{t}\left(r_{1}, \cdots, r_{t+1}\right)$ to prove the following formulae.

(3) $F\left(r_{1}\right)=1$,

(4) $\quad F_{1}\left(r_{1}, r_{2}\right)=\left(2^{r_{1}}-1\right)^{r_{2}}$,

(5) $F_{2}\left(r_{1}, 1, r_{3}\right)=\sum_{l=1}^{r_{1}}\left(\begin{array}{c}r_{1} \\ l\end{array}\right) 2^{\left(r_{1}-l\right) r_{3}}$,

(6) $\quad F_{2}\left(1, r_{1}, r_{2}\right)=\sum_{l=1}^{r_{1}} \sum_{m=1}^{r_{2}} 2^{r_{2}-m}\left(\begin{array}{c}r_{1} \\ l_{1}\end{array}\right)\left(\begin{array}{c}r_{2} \\ m\end{array}\right)\left(2^{r_{2}-1}\right)^{r_{1}-l}\left\{\left(2^{m}-1\right)^{l}-m\left(2^{m-1}-1\right)^{l}\right\}$,

(7) $F_{t}\left(1,1, \cdots, 1, r_{t+1}\right)=\sum_{j_{1}>0, j_{1}+\cdots+j_{t} \leqq r_{t+1}}\left(\begin{array}{c}r_{t+1} \\ j_{1}\end{array}\right)\left(\begin{array}{c}r_{t+1}-j \\ j_{2}\end{array}\right) \cdots$

$$
\cdots\left(\begin{array}{c}
r_{t+1}-\left(j_{1}+\cdots+j_{t-1}\right) \\
j_{t}
\end{array}\right) \text {. }
$$

1 Strictly speaking, members of $B_{4-i}$ must be taken as the unions $\cup Y(i, k)$ of all sets represented by the $x$ 's in $Y(i, k)$, but since $x$ 's represent disjoint sets this will not effect our conclusion about $F_{3}\left(r_{1}, \cdots, r_{4}\right)$. 
As an illustration we prove (5). We have to consider the number of ways some of the $x(i, j)$ can be placed in the empty squares in fig. 2 below such that (iv), (v) are satisfied.

\begin{tabular}{|c|c|c|c|c|c|}
\hline \multirow[t]{2}{*}{$x(1,1)$} & $x\left(1, e_{1}\right)$ & & & & $e_{I}=r_{2}$ \\
\hline & & $x\left(2, e_{t}+1\right)$ & & & $e_{2}=r_{2}+1$ \\
\hline & & & $x\left(3, e_{2}+1\right)$ & $x\left(3, e_{3}\right)$ & $e_{3}$ \\
\hline
\end{tabular}

Figure 2

In every empty square of the second row of this figure we must put just $x\left(2, e_{1}+1\right)$. In the square $\sigma\left(3, e_{1}+1\right)$ under $x\left(2, e_{1}+1\right)$ we can place any subset $\Sigma\left(3, e_{2}+1\right)$ of $\left\{x\left(3, e_{2}+1\right), \cdots, x\left(3, e_{3}\right)\right\}$. In the remaining empty squares of the third row we must put every symbol in $\Sigma\left(3, e_{2}+1\right)$ in addition to some other symbols arbitrarily selected from

$$
\left\{x\left(3, e_{2}+1\right), \cdots,\left(3 e_{3}\right)\right\}-\Sigma\left(3, e_{2}+1\right) .
$$

The formula (5) is now obvious.

We have employed formulae $(1)-(7)$ in calculating $N(n)$ for $n=3,4,5,6$.

In the end I would like to thank Professor Hanna Neumann for her useful suggestions for the improvement in the presentation of the material of this paper. My thanks are also due to the referee for his very valuable criticism.

Institute of Advanced Studies

The Australian National University

Canberra, A.C.T.

and

Panjab University

Lahore, Pakistan 